\title{
Non-Finite Axiomatizability and Undecidability of Interval Temporal Logics with C, D, and T
}

\author{
Ian Hodkinson ${ }^{1}$, Angelo Montanari ${ }^{2}$, and Guido Sciavicco ${ }^{3}$ \\ 1 Department of Computing, Imperial College London, \\ South Kensington Campus London SW7 2AZ (UK), imh@doc.ic.ac.uk \\ 2 Department of Mathematics and Computer Science, \\ University of Udine (Italy), angelo.montanari@dimi.uniud.it \\ 3 Department of Information Engineering and Communications, \\ University of Murcia, Murcia (Spain), guido@um.es
}

\begin{abstract}
Interval logics are an important area of computer science. Although attention has been mainly focused on unary operators, an early work by Venema (1991) introduced an expressively complete interval logic language called CDT, based on binary operators, which has many potential applications and a strong theoretical interest. Many very natural questions about CDT and its fragments, such as (non-)finite axiomatizability and (un-)decidability, are still open (as a matter of fact, only a few undecidability results, including the undecidability of CDT, are known). In this paper, we answer most of these questions, showing that almost all fragments of CDT, containing at least one binary operator, are neither finitely axiomatizable with standard rules nor decidable. A few cases remain open.
\end{abstract}

\section{Introduction}

Interval-based temporal logic represents an important area of computer science. The main species of propositional interval temporal logics studied so far include Mozskowski's Propositional Interval Logic (PITL) [22], Halpern and Shoham's Modal Logic of Allen's Relations (HS) [16], and Venema's CDT logic [25] (extended to branching-time frames with linear intervals in [14]). Important fragments of HS studied in more detail include, among others, the logic of begins/ends Allen's relations (BE) [19], the logics of temporal neighborhood $[8,9,12]$ and the logics of subinterval structures $[4,5]$.

(Un-)decidability. The logic PITL, which features the binary modality $C$ (chop) and the modal constant $\pi$ for point-intervals, has been shown to be undecidable in [22] when interpreted in the class of all finite linearly ordered sets and in classes based on $\mathbb{N}$ or $\mathbb{Z}$; in [19] the result has been extended to the dense case (as pointed out in [13], this implies undecidability in the class of all linearly ordered sets). Satisfiability for HS is also undecidable in many cases, as it has been shown in [16]. HS can be thought of as a modal logic with a unary modal operator for any of Allen's relations between two intervals. Undecidability has 
been shown for various interesting classes of linearly ordered sets, and the proof hinges on a non-trivial reduction from the halting problem for a Turing machine. In some cases, such as Dedekind-complete linearly ordered sets with an infinite unbounded ascending sequence, validity is non recursively enumerable, which implies non-finite axiomatizability (no matters what kind of deduction rules are used). Undecidability of CDT, over the same classes of structures, immediately follows from that of HS. In [19], the fragment of HS with only two modalities, corresponding to Allen's relations begins/ends (BE), has been shown undecidable when interpreted over dense linear orderings. On the positive side, decidability of the fragments of HS with modalities corresponding to Allen's relations begins/begun-by and ends/ended-by has been obtained by means of a translation to Linear Temporal Logic [13]. Moreover, decidability of the satisfiability problem for the class of HS fragments featuring only two modalities, corresponding to Allen's relations meets/met-by (A $\overline{\mathrm{A}}$, or $\mathrm{PNL}$ ), has been shown in [6] (as a matter of fact, a number of natural extensions of it turn out to be undecidable $([7]))$. Finally, decidability of the logics of subinterval structures over dense ordering has been proved in $[4,5]$.

(Non-)finite axiomatizability. Sound and complete axiomatic systems in interval temporal logics are scarce. PITL has been axiomatized both at the firstorder and the propositional level in [3] for discrete/finite linearly ordered sets, but under the hypothesis of locality, that is, with the semantical assumption that each propositional letter is true over an interval if and only if it is true at the first point of the interval. The logics HS and CDT have been finitely axiomatized, respectively in [24] and [25], for various classes of linearly ordered sets, but using a Burgess/Gabbay-style non-orthodox 'irreflexivity' rule [11]. Finally, logics of neighborhood modalities have complete axiomatic systems with standard rules in various cases; some of them are infinite, as in the case in which point-intervals are allowed, but no modal constant for them is included in the language [12].

In this paper we focus on the logic CDT. Its language includes three binary operators $C, D$, and $T$, which correspond to the ternary interval relations occurring when an extra point is added in one of the three possible distinct positions with respect to the two endpoints of the current interval (between, before, and after), plus a modal constant $\pi$ which holds at a given interval if and only if it is a point-interval. We prove the following results. First, we show that the undecidability of CDT can be extended to some sub-languages. For the language with $C$ and $\pi$, undecidability was known (as recalled above). Here we show that $D$ alone and $T$ alone are undecidable as well, when interpreted in interval structures over any class of linearly ordered sets containing at least one linearly ordered set with an infinite sequence - ascending for $T$, descending for $D$ - and that $D+\pi$ and $T+\pi$ are undecidable when interpreted in interval structures over finite strict linear orders. Second, the logic CDT is not finitely axiomatizable with standard rules over interval structures based on any class of linearly ordered sets containing $(\mathbb{Q},<)$. The same holds for any sub-language containing at least one of the modalities $C, D, T$. This result holds even if we drop the modal constant $\pi$. Notice that the undecidability and non-finite axiomatizability results are inde- 
pendent from each other. The question of whether there exists a finite axiomatic system for CDT with standard rules only was open since [24,25]. The results in the present paper correct a previous claim in [23]. Because of space restrictions, many proofs are only sketched in bare outline or omitted.

\section{Basic Notions}

Venema's interval-based temporal logic CDT [25] features a denumerable set $\mathcal{A P}$ of propositional letters, the classical operators $\wedge$ and $\neg$ (the remaining ones can be considered as abbreviations), the modal constant $\pi$, and three binary modal operators, namely, $C, T$, and $D .{ }^{4}$ Well-formed formulas, denoted by $\phi, \psi, \ldots$, can be obtained by the following grammar:

$$
\phi:=p|\neg \phi| \phi \wedge \psi|\pi| \phi \sharp \psi(\sharp \in \operatorname{Mod}),
$$

where $\operatorname{Mod}=\{C, D, T\}$ and $p \in \mathcal{A} \mathcal{P}$. We will denote the sublanguage of CDT featuring only the non-empty set $S \subseteq \operatorname{Mod}$ of the operators by $L_{S}^{\pi}$, and by $L_{S}$ when $\pi$ is not allowed (so CDT becomes $L_{C D T}^{\pi}$ in our notation). In the next section, we will show that, for every $S, L_{S}$ is not expressive enough to define $\pi$.

Definition 1 (abstract semantics). Let $S \subseteq$ Mod. An $S$-frame is a structure of the form $\mathcal{F}=\left(I, \Pi, R_{\sharp}: \sharp \in S\right)$, where $I$ is a non-empty set, $\Pi \subseteq I$, and $R_{\sharp} \subseteq I^{3}$ for each $\sharp \in S$. An $S$-model is a structure of the form $\mathcal{M}=(\mathcal{F}, h)$, where $\mathcal{F}=\left(I, \Pi, R_{\sharp}: \sharp \in S\right)$ is an $S$-frame and $h: \mathcal{A P} \rightarrow \mathcal{P}(I)$. The frame of $\mathcal{M}$ is $\mathcal{F}$. We evaluate an $L_{S}^{\pi}$-formula $\phi$ in $\mathcal{M}$ at $i \in I$ as follows:

- $\mathcal{M}, i \models p$ iff $i \in h(p)$, for $p \in \mathcal{A P}$;

$-\mathcal{M}, i \models \neg \phi$ iff it is not the case that $\mathcal{M}, i \models \phi$;

- $\mathcal{M}, i \models \phi \wedge \psi$ iff $\mathcal{M}, i \models \phi$ and $\mathcal{M}, i \models \psi$;

$-\mathcal{M}, i=\pi$ iff $i \in \Pi$;

- $\mathcal{M}, i \models \phi \sharp \psi$ iff there exist $j, k \in I$ with $R_{\sharp}(i, j, k), \mathcal{M}, j=\phi$, and $\mathcal{M}, k=\psi$.

Throughout, we often identify (notationally) a model or frame with its domain: so, for example, we write $i \in \mathcal{F}$ or $i \in \mathcal{M}$ to mean $i \in I$ as above. Let $S \subseteq$ Mod, $\mathcal{M}$ be an $S$-model, and $\mathcal{K}$ be a class of $S$-frames. An $L_{S}^{\pi}$-formula $\phi$ is said to be satisfiable in $\mathcal{M}$ if there is some $i \in \mathcal{M}$ such that $\mathcal{M}, i \models \phi$, and satisfiable over $\mathcal{K}$ if it is satisfiable in a model whose frame is in $\mathcal{K}$. Similarly, an $L_{S}^{\pi}$ formula $\phi$ is valid in $\mathcal{M}$ (resp., valid over $\mathcal{K}$ ) if for every $i \in \mathcal{M}$, it is the case that $\mathcal{M}, i \models \phi$ (resp., if $\phi$ is valid in every model whose frame is in $\mathcal{K}$ ). These definitions naturally generalize to the case of sets of formulas.

Notice that $L_{S}$-formulas are $L_{S}^{\pi}$-formulas, so we can evaluate them in $S$-models. We do not need to include $\Pi$ in our frames in this case, but it can always be added without affecting $L_{S}$-semantics. So rather than to complicate our notation by introducing frames without $\Pi$, we give $L_{S}$-formulas semantics in $L_{S}^{\pi}$-models, which include $\Pi$.

While the above semantics can be considered abstract, we can formalize a concrete one based on pairs of points (intervals) over linear orders.

\footnotetext{
${ }^{4} C$ stands for 'chop'. One may think of $D$ as standing for 'Done' and $T$ for 'To come'.
} 


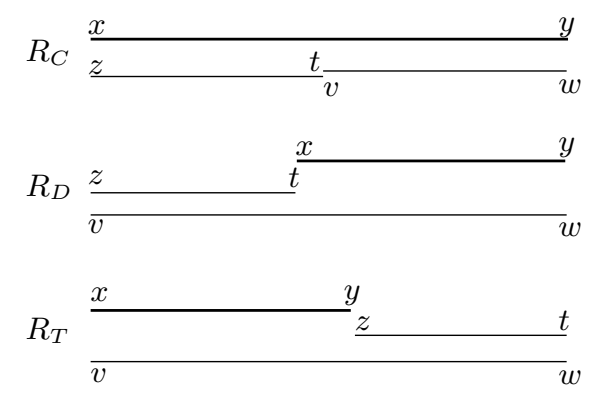

Fig. 1. The relations $R_{C}, R_{D}, R_{T}$; the thick line represents the current interval $[x, y]$.

Definition 2 (concrete semantics). Let $(T,<)$ be a strict linear order. We write $\operatorname{Int}(T,<)$ for the set $\{[t, u]: t, u \in T, t \leq u\}$. As usual, $[t, u]=\{v \in T$ : $t \leq v \leq u\}$, and $t \leq u$ abbreviates $t<u \vee t=u$. Let $\Pi=\{[t, t]: t \in T\}$. For any $[x, y],[z, t],[v, w] \in \operatorname{Int}(T,<)$, we define

$-R_{C}([x, y],[z, t],[v, w])$ iff $x=z, t=v$, and $y=w$;

$-R_{D}([x, y],[z, t],[v, w])$ iff $x=t, z=v$, and $y=w$;

$-R_{T}([x, y],[z, t],[v, w])$ iff $x=v, y=z$, and $t=w$.

For $S \subseteq M o d$, we write $\mathfrak{I n t}_{S}(T,<)$ for the $S$-frame $\left(\operatorname{Int}(T,<), \Pi, R_{\sharp}: \sharp \in S\right)$.

A graphical account of the three relations is given in Fig. 1. Intuitively, for an interval $[x, y]$, we have $\mathcal{M},[x, y] \models \phi C \psi$ iff there is $z \in[x, y]$ with $\mathcal{M},[x, z] \models$ $\phi$ and $\mathcal{M},[z, y]=\psi$, and similarly for the other two modalities. We will use alternatively the abstract or the concrete semantics.

Definition 3. Let $S \subseteq$ Mod and let $\mathcal{K}$ be a class of strict linear orders. We write $S(\mathcal{K})$ (resp., $S^{\pi}(\mathcal{K})$ ) for the set of all $L_{S}$-formulas (resp., $L_{S}^{\pi}$-formulas) valid over $\left\{\mathfrak{I n t}_{S}(T,<):(T,<) \in \mathcal{K}\right\}$. Thus, $S(\mathcal{K})$ and $S^{\pi}(\mathcal{K})$ are the logics of (intervals over) $\mathcal{K}$ in their respective languages.

Beside the usual $\mathbb{N}, \mathbb{Z}, \mathbb{Q}$, we introduce notation for some common classes:

- Lin $=$ the class of all strict linear orders

- Fin $=$ the class of all finite strict linear orders

- Dense $=$ the class of all strict dense linear orders

- Dis = the class of all strict discrete linear orders

- Asc $=$ the class of all strict linear orders that contain an infinite ascending sequence (i.e., they have $(\mathbb{N},<)$ as a suborder)

- Des $=$ the class of all strict linear orders that contain an infinite descending sequence

We usually write $S \subseteq \operatorname{Mod}$ as a sequence consisting of its members: thus, we have the logics $C D T($ Lin $), C^{\pi}$ (Dense), etc. When $\mathcal{K}=\{(T,<)\}$, we write $S(\mathcal{K})$ as $S(T,<)$ and $S^{\pi}(\mathcal{K})$ as $S^{\pi}(T,<)$.

\section{Expressive Power of $L_{C D T}$ and $L_{C D T}^{\pi}$}

While in this paper we are mainly interested in binary modalities, unary modalities are most studied in interval logics. In general, one can introduce a unary 
interval modality for each of Allen's relations between two intervals. In [16] it was shown that only four of them are sufficient to express any of Allen's relations between two intervals, namely, $\langle B\rangle$ (begins), $\langle E\rangle$ (ends) and their inverses. In the language $L_{C D T}^{\pi}$ these operators can be easily expressed, e.g.: $\langle B\rangle \phi=\phi C \neg \pi$, or $\langle\bar{E}\rangle \phi=\neg \pi D \phi$. In [24] the undecidability of $C D T$ (Asc) (and, by symmetry, of $C D T($ Des $)$ ) has been proved by exploiting the undecidability of HS over the same classes. Notice that the modal constant $\pi$ plays an important role here. So, the question is whether $\pi$ is definable in the language $L_{C D T}$ or not. Here we show that it is not the case, proving that $L_{C D T}$ is strictly less expressive than $L_{C D T}^{\pi}$, by applying a simple bisimulation argument to exhibit two models that can be distinguished by an $L_{C D T}^{\pi}$-formula, but not by any $L_{C D T}$-formula.

Theorem 1. The modal constant $\pi$ cannot be defined in $L_{C D T}$.

Proof. Consider the pair of models $\left(\mathcal{M}_{0}, \mathcal{M}_{1}\right)$, based, respectively, on $\operatorname{Int}\left(T_{0},<\right)$ and $\operatorname{Int}\left(T_{1},<\right)$, where $T_{0}=\left\{x_{0}, z_{0}, y_{0}\right\}$ with $x_{0}<z_{0}<y_{0}$, and $T_{1}=\left\{x_{1}, y_{1}\right\}$ with $x_{1}<y_{1}$. Clearly, $\operatorname{Int}\left(T_{0},<\right)=\left\{\left[x_{0}, x_{0}\right],\left[y_{0}, y_{0}\right],\left[z_{0}, z_{0}\right],\left[x_{0}, y_{0}\right],\left[x_{0}, z_{0}\right]\right.$, $\left.\left[z_{0}, y_{0}\right]\right\}$, and $\operatorname{Int}\left(T_{1},<\right)=\left\{\left[x_{1}, x_{1}\right],\left[y_{1}, y_{1}\right],\left[x_{1}, y_{1}\right]\right\}$. The valuation functions $h_{0}$ and $h_{1}$ are void. It is easily seen that the intervals $\left[x_{0}, z_{0}\right]$ and $\left[x_{1}, x_{1}\right]$ are bisimilar. By the bisimulation-invariance of modal formulas (see, e.g., [1, Theorem 2.20]), they satisfy the same $L_{C D T}$-formulas in their respective models. But $\mathcal{M}_{0},\left[x_{0}, z_{0}\right] \models \neg \pi$ and $\mathcal{M}_{1},\left[x_{1}, x_{1}\right] \models \pi$.

Because $\pi$ is equivalent to $[B] \perp$ (and to $[E] \perp$ ), this means that $L_{C D T}$ cannot define all of the modalities corresponding to Allen's relations, and the original argument for the undecidability cannot be applied anymore. As for fragments of $L_{C D T}^{\pi}$, the logic $C^{\pi}$ has been shown to be undecidable for discrete linear orders in [22]; later, in [19], the undecidability was shown also for dense orders, and thus, since density and the universal operator can be defined in the language (see [13]), for all linear orders. The other fragments, with or without $\pi$, have received no attention so far.

\section{Undecidability}

In this section, we establish undecidability of any logic $D(\mathcal{K})$ where $\mathcal{K} \subseteq$ Lin and $\mathcal{K} \cap \operatorname{Des} \neq \emptyset$, and $T(\mathcal{K})$, where $\mathcal{K} \subseteq$ Lin and $\mathcal{K} \cap$ Asc $\neq \emptyset$. Recall that Asc (resp., Des) is the class of all linearly ordered sets with an infinite ascending (resp., descending) sequence. We consider the case of $T$, the other one is symmetric. We show how to encode in $L_{T}$ a variant of the $\mathbb{N} \times \mathbb{N}$-tiling problem called the Octant Tiling Problem. Given a set of tiles $\mathcal{T}=\left\{t_{1}, \ldots, t_{k}\right\}$, the octant tiling problem consists in establishing whether $\mathcal{T}$ can tile one octant of the Cartesian plane over $\mathbb{N}$; in our case, it will be the second octant $\mathcal{O}=\{(p, q) \mid p, q \in \mathbb{N}, p \leq q\}$. Each tile $t_{i}$ has four colors, namely, right $\left(t_{i}\right)$, left $\left(t_{i}\right), u p\left(t_{i}\right)$, and $\operatorname{down}\left(t_{i}\right)$, and neighboring tiles must have matching colors. Formally, we say that a set $\mathcal{T}$ can tile $\mathcal{O}$ if there exists a function $f: \mathcal{O} \mapsto \mathcal{T}$ such that $\operatorname{right}(f(p, q))=$ left $(f(p+1, q))$ and $u p(f(p, q))=\operatorname{down}(f(p, q+1))$, where $f(p, q)$ represents 
the tile to be placed in the position $(p, q)$, whenever all relevant coordinates $((p, q),(p+1, q)$ etc. $)$ lie in $\mathcal{O}$. The undecidability of the $\mathbb{N} \times \mathbb{N}$-tiling problem is well-known (e.g., see [2]); one can easily prove that the octant tiling problem is undecidable as well.

\subsection{Language, Shortcuts, and $u$-Intervals}

Let $\mathcal{T}=\left\{t_{1}, \ldots, t_{k}\right\}$ be an instance of the octant tiling problem. We will assume that $\mathcal{A} P$ contains at least the propositional letters $u, t_{1}, \ldots$, and $t_{k}$.

As a preliminary step, we introduce a sort of universal operator (denoted by $G$ ) that looks only at the future of the current interval, and, then, we set our framework by forcing the existence of unit-intervals (or u-intervals) working like atomic elements. Such intervals will be denoted by the propositional letter $u$. We will impose that $u$-intervals are disposed in an unbounded unique (uninterrupted) sequence:

$$
\begin{gathered}
G \phi::=\neg(\top T(\neg \phi T \top)), \\
u T \top \wedge G(u \rightarrow u T \neg u) .
\end{gathered}
$$

It is not difficult to see that $\mathcal{M},[x, y] \models G \phi$ iff $\forall z, t(y \leq z \leq t \rightarrow \mathcal{M},[z, t] \models \phi)$. Lemma 1. Suppose that $\mathcal{M}$ is a model with frame $\mathfrak{I n t}_{T}(T,<)$ for any strict linear order $(T,<)$, that $[x, y] \in \mathcal{M}$, and that $\mathcal{M},[x, y] \models(2)$. Then, there exists an infinite sequence of points $y_{0}<y_{1}<\cdots$ such that

1. $y=y_{0}$;

2. For every $l \in \mathbb{N}, \mathcal{M},\left[y_{l}, y_{l+1}\right] \models u$.

\subsection{The Encoding of the Tiling Problem and Undecidability}

Since the set of tiles $\mathcal{T}$ is finite, the set of colors is finite as well. Let us define an arbitrary order over it and denote the $i$-th color by $\operatorname{col}(i)$. The propositional letters $t_{1}, \ldots, t_{k}$ are used to encode the colors of each side of the corresponding tiles $t_{1}, \ldots, t_{k}$, that is, to state that $\operatorname{right}\left(t_{i}\right)=\operatorname{color}\left(k_{r}\right), \operatorname{left}\left(t_{i}\right)=\operatorname{color}\left(k_{l}\right)$, up $\left(t_{i}\right)=\operatorname{color}\left(k_{u}\right)$, and down $\left(t_{i}\right)=\operatorname{color}\left(k_{d}\right)$, for suitable $k_{r}, k_{l}, k_{u}$, and $k_{d}$.

The next formulas state that all $u$-intervals are tiles and that, whenever a tile is placed, it is unique. Moreover, they guarantee that tiles are placed in such a way that they respect conditions on colors.

$$
\begin{array}{r}
G\left(u \rightarrow \bigvee_{i=1}^{|\mathcal{T}|} t_{i}\right), \\
G \bigwedge_{i=1}^{|\mathcal{T}|} \neg\left(t_{i} \wedge t_{j}\right), \\
G\left(u \rightarrow t_{i \neq j} \rightarrow\left(u T \neg \bigwedge_{j=1, u p\left(t_{i}\right)=\operatorname{down}\left(t_{j}\right)}^{|\mathcal{T}|} t_{j}^{|\mathcal{T}|} \bigwedge_{i=1, j=1, \text { right }\left(t_{j}\right) \neq \operatorname{left}\left(t_{i}\right)} \neg\left(t_{i} T t_{j}\right)\right),\right.
\end{array}
$$




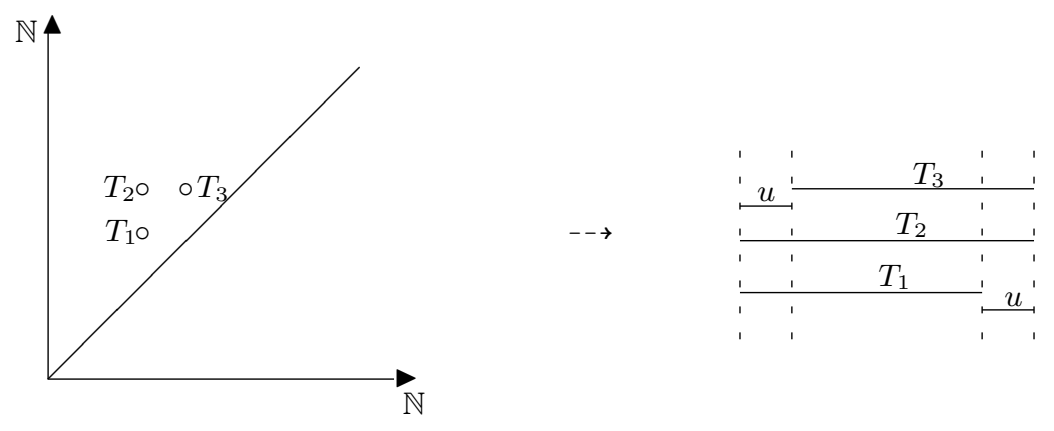

Fig. 2. A pictorial representation of the encoding.

Now we define

$$
\phi_{\mathcal{T}}=(2) \wedge(3) \wedge(4) \wedge(5) \wedge(6) .
$$

Lemma 2 (soundness). Let $\mathcal{T}=\left\{t_{1}, \ldots, t_{k}\right\}$ be a set of tiles. If $\phi_{\mathcal{T}}$ is satisfiable, then $\mathcal{T}$ tiles the second octant $\mathcal{O}$.

Proof. Let $\mathcal{M},[x, y] \models \phi_{\mathcal{T}}$. We show that there exists a tiling function $f: \mathcal{O} \mapsto$ $\mathcal{T}$. By Lemma 1, we know that there exists a unbounded sequence of points $y_{0}<y_{1}<\ldots$ such that $y=y_{0}$, and, for every $l \in \mathbb{N}, \mathcal{M},\left[y_{l}, y_{l+1}\right] \models u$. Now, for each $l, m \in \mathbb{N}, l \leq m$, we put:

$$
f(l, m)=t \text {, where } \mathcal{M},\left[y_{l}, y_{m+1}\right] \models t .
$$

First, we have to show that $f$ is well-defined, that is, that each $f(l, m)$ is a tile. We proceed by induction on $(m-l)$. If $(m-l)=0$, then we are on a $u$-interval, and, by (3), it must be a tile. If more than one tile is placed over, it suffices to choose one of them randomly, and thus $f$ is a function. Suppose now that $f(l, m)$ is a tile whenever $m-l \leq p$, and consider $m-l=p+1$. Since $((m-1)-l) \leq p$, by inductive hypothesis $f(l, m-1)$ is a tile, say $t_{i}$. This means that $\mathcal{M},\left[y_{l}, y_{m}\right] \models t_{i}$, and, by $(5), \mathcal{M},\left[y_{l}, y_{m}\right] \models \neg\left(u T \neg \bigvee_{u p\left(t_{i}\right)=\operatorname{down}\left(t_{j}\right)} t_{j}\right)$. So, for every $y \geq y_{m}$, if $\mathcal{M},\left[y_{m}, y\right] \models u$, it must be the case that $\mathcal{M},\left[y_{l}, y\right] \models \bigvee_{u p\left(t_{i}\right)=\operatorname{down}\left(t_{j}\right)} t_{j}$. This applies to the particular case $y=y_{m+1}$, since $\mathcal{M},\left[y_{m}, y_{m+1}\right] \models u$. Hence, we have that $\mathcal{M},\left[y_{l}, y_{m+1}\right] \models t_{j}$, that is, $f(l, m)=t_{j}$, for some $j$ such that down $\left(t_{j}\right)=u p\left(t_{i}\right)$. This guarantees us that $f$ respects the 'vertical' condition of a tiling function. To conclude the proof, we need to show that that the 'horizontal' condition is respected as well. To this end, consider $f(l, m)$ and $f(l+1, m)$. By definition, the corresponding tiles are $\left[y_{l}, y_{m+1}\right]$ and $\left[y_{l+1}, y_{m+1}\right]$. Since, by definition, the interval $\left[y_{l}, y_{l+1}\right]$ is a $u$-interval, by (6) it cannot be the case that left $(f(l+1, m)) \neq \operatorname{right}(f(l, m))$, which implies that $\operatorname{left}(f(l+1, m))=$ $\operatorname{right}(f(l, m))$.

Lemma 3 (completeness). Let $\mathcal{T}=\left\{t_{1}, \ldots, t_{k}\right\}$ be a set of tiles and $f: \mathcal{O} \mapsto$ $\mathcal{T}$ be a tiling function. Then $\phi_{\mathcal{T}}$ is satisfiable over (the intervals of) any linearly ordered set with an infinite ascending sequence. 
Proof. Assuming for simplicity that the order is $(\mathbb{N},<)$, one can make $\phi_{\mathcal{T}}$ true at $[0,0]$ by letting $u$ be true at all intervals of length 1 , and each $t_{i}$ be true at all intervals of the form $[x, y+1]$, where $f(x, y)=t_{i}$.

We can prove similar results for $S \subseteq\{C, D, T\}$ containing $C$, without using the modal constant $\pi$, over any $\mathcal{K} \subseteq$ Lin containing some $(T,<)$ that has an infinite interval $[x, y]$. The construction is analogous to the previous one. Assuming that $[x, y]$ contains an infinite increasing sequence, we substitute $T$ by $C$ in (1), and modify (2), (5), and (6), as follows:

$$
\begin{array}{r}
u C \top \wedge G(u \rightarrow \top C \neg u) \wedge \neg(\top C(u C \neg(u C \top))), \\
G \bigwedge_{i=1}^{|\mathcal{T}|}\left(t_{i} C u \rightarrow \bigvee_{j=1, u p\left(t_{i}\right)=\operatorname{down}\left(t_{j}\right)}^{|\mathcal{T}|} t_{j}\right), \\
G \bigwedge_{i=1}^{|\mathcal{T}|}\left(u C t_{i} \rightarrow \bigvee_{j=1, \operatorname{right}\left(t_{j}\right)=\operatorname{left}\left(t_{i}\right)} t_{j}\right) .
\end{array}
$$

If the resulting conjunction $\phi_{\mathcal{T}}$ is satisfiable over Lin, then $\mathcal{T}$ tiles the second octant; this implies that $\phi_{\mathcal{T}}$ is satisfiable on $[x, y]$. A mirror image formula works when $[x, y]$ contains an infinite descending sequence.

Theorem 2. For any $S \subseteq\{C, D, T\}$ containing $T$, the logics $S(\mathcal{K})$ and $S^{\pi}(\mathcal{K})$ are not decidable for any $\mathcal{K} \subseteq$ Lin with $\mathcal{K} \cap$ Asc $\neq \emptyset$, in particular when $\mathcal{K}$ is any of the following: Lin, Dense, Dis, Asc, the class of all infinite linearly ordered sets, any of the usual linear orders based on $\mathbb{N}, \mathbb{Q}, \mathbb{R}$, etc. The same applies for any $S \subseteq\{C, D, T\}$ containing $D$, substituting Asc with Des, and $\mathbb{N}$ with $\mathbb{Z}$ or $\mathbb{Z} \leq 0$. Finally, for any $S \subseteq\{C, D, T\}$ containing $C, S(\mathcal{K})$ and $S^{\pi}(\mathcal{K})$ are undecidable whenever $\mathcal{K} \subseteq$ Lin contains some $(T,<)$ such that some interval in $\operatorname{Int}(T,<)$ is infinite - e.g., when $\mathcal{K}$ is Lin, Asc, Des, Dense, Dis, $\{(\mathbb{Q},<)\}$, or $\{(\mathbb{R},<)\}$.

Proof. If $\mathcal{K}$ is any class with $\mathcal{K} \subseteq$ Lin and $\mathcal{K} \cap$ Asc $\neq \emptyset$, then $\phi_{\mathcal{T}}$ is satisfiable over $\mathcal{K}$ (and hence $\neg \phi_{\mathcal{T}} \notin T(\mathcal{K}), T^{\pi}(\mathcal{K})$ ) iff $\mathcal{T}$ tiles $\mathcal{O}$. Since $\phi_{\mathcal{T}}$ can be constructed from $\mathcal{T}$ by an algorithm, the result for $T$ follows from the undecidability of the octant tiling problem. The other cases are similar.

\subsection{Undecidability over finite models}

The above argument cannot be applied when we interpret the language over finite models. On the other hand, in [22] the language $L_{C}^{\pi}$ has been shown to be undecidable when interpreted in the class of all finite linearly ordered sets, or, equivalently (since this logic can only 'look' inside the initial interval), over $\mathbb{N}$ or $\mathbb{Z}$. Here we show how to adapt the same argument to show that the logics $D^{\pi}$ (Fin) and $T^{\pi}$ (Fin) are undecidable too; again, we focus on the latter only. We closely follow [22]. In more detail, let $\mathcal{G}$ be a context-free grammar in Greibach normal form with terminals 0,1 , say, and set of non-terminals $\mathcal{N}(\mathcal{G})=\left\{A_{0}, \ldots, A_{N}\right\}$, say, where $A_{0}$ is initial. We assume that the language generated by $\mathcal{G}$ does not 
contain the empty string $\varepsilon$, and hence that $\mathcal{G}$ has no productions of the form $A_{i} \longrightarrow \varepsilon$. So all productions are of the form $A_{i} \longrightarrow V_{1} V_{2} \ldots V_{m}$, where $m \geq 1$, $V_{1}$ is a terminal, and $V_{2}, \ldots, V_{m}$ are terminals or non-terminals.

Whenever a word $w_{0} w_{1} \ldots w_{n-1}$ is generated by $\mathcal{G}$, there is a finite derivation tree $D$ for it (and conversely). Each node $d$ of $D$ has a label $\lambda(d)$ : it is 0 or 1 if $d$ is a leaf, and some non-terminal $A_{i}$ otherwise, in which case the children of $d$ taken in left-right order are labeled $V_{1}, V_{2}, \ldots, V_{m}$, respectively, for some production rule $A_{i} \longrightarrow V_{1} V_{2} \ldots V_{m}$ of $\mathcal{G}$ ( $V_{1}$ is always a terminal and so the leftmost child of any node is a leaf). The root of $D$ is labeled $A_{0}$, and the leaves taken in left-right order are labeled $w_{0}, w_{1}, \ldots, w_{n-1}$, respectively.

We regard each non-terminal as a propositional letter, and we also use two more propositional letters, $p$ and $u$. Thus, our set of propositional letters is $\mathcal{L}(\mathcal{G}):=\mathcal{N}(\mathcal{G}) \cup\{p, u\}$. We let $\phi_{\mathcal{G}}$ be the conjunction of the following $L_{T^{-}}^{\pi}$ formulas, where $G \phi$ is defined as in (1), and $A_{0}$ is the starting symbol for $\mathcal{G}$ :

$$
\begin{aligned}
& G(u \rightarrow \neg \pi) \wedge G \neg(\neg \pi \wedge(\neg \pi T u)), \\
&\left(A_{0} \wedge \neg(\neg \pi T \top)\right) T \top, \\
& G \neg\left(A_{i} \wedge\left(\neg \pi T A_{i}\right)\right), \forall i=0, \ldots, N, \\
&\left.G\left(\pi \wedge A_{i} T \top \rightarrow \bigvee_{j=1}^{n(i)} \widehat{V_{1}^{j}} T \widehat{\left(V_{2}^{j}\right.} T\left(\cdots T\left(\widehat{V_{m(i, j)}^{j}} T A_{i}\right)\right) \cdots\right)\right), \forall i=0, \ldots, N,
\end{aligned}
$$

where

$$
A_{i} \longrightarrow V_{1}^{1} V_{2}^{1} \ldots V_{m(i, 1)}^{1}\left|V_{1}^{2} V_{2}^{2} \ldots V_{m(i, 2)}^{2}\right| \cdots \mid V_{1}^{n(i)} V_{2}^{n(i)} \cdots V_{m(i, n(i))}^{n(i)}
$$

is the 'consolidated' production rule of $\mathcal{G}$ for $A_{i}$, and for any terminal or nonterminal $V$ :

$$
\widehat{V}= \begin{cases}u \wedge p, & \text { if } V=1, \\ u \wedge \neg p, & \text { if } V=0, \\ V, & \text { otherwise. }\end{cases}
$$

Lemma 4 (soundness). Let $\mathcal{G}_{1}, \mathcal{G}_{2}$ be grammars as above, with (wlog.) $\mathcal{N}\left(\mathcal{G}_{1}\right) \cap$ $\mathcal{N}\left(\mathcal{G}_{2}\right)=\emptyset$. Note that $\mathcal{L}\left(\mathcal{G}_{1}\right) \cap \mathcal{L}\left(\mathcal{G}_{2}\right)=\{p, u\}$. If the $L_{T}^{\pi}$-formula $\psi=\phi_{\mathcal{G}_{1}} \wedge$ $\phi_{\mathcal{G}_{2}}$ is satisfiable in a model with frame of the form $\mathfrak{I n t}_{T}(T,<)$ for some finite strict linear order $(T,<)$, then the languages generated by $\mathcal{G}_{1}, \mathcal{G}_{2}$ have a word in common.

Proof. Suppose that $\psi$ is satisfiable in a model $\mathcal{M}$ with frame $\mathfrak{I n t}_{T}(T,<)$, where $(T,<) \in$ Fin. We can suppose wlog. that $\mathcal{M},[0,0] \models \psi$ for some point $0 \in T$, where $\{x \in T: x \geq 0\}=\{0,1, \ldots, n\}$ for some natural number $n \geq 0$, and $<$ is the usual ordering on this set. We will see below that $n>0$. For $x<n$ put

$$
w_{x}= \begin{cases}1, & \text { if } \mathcal{M},[x, x+1] \models p \\ 0, & \text { otherwise }\end{cases}
$$


We show that the word $w=w_{0} \ldots w_{n-1}$ is generable by $\mathcal{G}_{1}$ by constructing a derivation tree $D$ for it. Each node $d$ of $D$ will be associated with an interval $\left[x_{d}, y_{d}\right]$ of $(T,<)$, where $0 \leq x_{d} \leq y_{d}$. We will add nodes $d$ to $D$ step by step, in such a way that

$$
\mathcal{M},\left[x_{d}, y_{d}\right]=\widehat{\lambda(d)} .
$$

Given this, if $d \in D$ is labeled by a terminal $\lambda(d)=\alpha \in\{0,1\}$, then $\mathcal{M},\left[x_{d}, y_{d}\right]=$ $\widehat{\alpha}$; by $(15), \widehat{\alpha} \vdash u$, so $\mathcal{M},\left[x_{d}, y_{d}\right] \models u$. As $\mathcal{M},[0,0] \models(11)$, we have $y_{d}=x_{d}+1$.

First we add the root $r$ to $D$, and put $\left[x_{r}, y_{r}\right]=[0, n]$ and $\lambda(r)=A_{0}$. As $\mathcal{M},[0,0] \models(12)$, we have $\mathcal{M},[0, n] \models A_{0}$, so (17) holds. Then we repeat the following while $D$ has leaves labeled by non-terminals. Pick such a leaf $d$, where $\lambda(d)$ is a non-terminal $A_{i}$, say. By $(17), \mathcal{M},\left[x_{d}, y_{d}\right] \models A_{i}$, so $\mathcal{M},\left[x_{d}, x_{d}\right] \models$ $\pi \wedge A_{i} T \top$. Since $\mathcal{M},[0,0] \models(14)$, there exist a production rule $A_{i} \longrightarrow V_{1} V_{2} \ldots V_{m}$ of $\mathcal{G}_{1}$ and points $x_{d}=x_{0} \leq x_{1} \leq \ldots \leq x_{m} \leq n$ with $\mathcal{M},\left[x_{j-1}, x_{j}\right] \models \widehat{V}_{j}$ for each $j=1, \ldots, m$, and $\mathcal{M},\left[x_{d}, x_{m}\right] \models A_{i}$. But $\mathcal{M},[0,0] \models(13)$, so $x_{m}=y_{d}$. We now add new nodes $d_{1}, \ldots, d_{m}$ to $D$ as the left-to-right children of $d$, and we put $\left[x_{d_{j}}, y_{d_{j}}\right]=\left[x_{j-1}, x_{j}\right]$ and $\lambda\left(d_{j}\right)=V_{j}$ for each $j=1, \ldots, m$. By choice of the $x_{j},(17)$ is preserved. Note that since $V_{1}$ is a terminal, $x_{d_{1}}<y_{d_{1}}$. It follows that $x_{d}<y_{d}$ for each $d \in D ; n>0$; and the lengths of intervals associated with non-terminals strictly decrease as we move from the root. So as $T$ is finite, the process terminates. Let $l_{0}, \ldots, l_{m-1}$ be the leaves of the final $D$ in left-to-right order. Each $l_{i}$ is labeled by a terminal, and so is associated with a two-point interval. Now the construction preserves the property that for any $x<n$, there is always a leaf of $D$ whose associated interval contains $[x, x+1]$. So at the end, this is still true, and it follows that $[x, x+1]$ is associated with some $l_{i}$. Therefore, $m=n$ and $l_{0}, \ldots, l_{n-1}$ are associated with $[0,1],[1,2], \ldots,[n-1, n]$, respectively. For each $x<n$ we have $w_{x}=1$ iff $\mathcal{M},[x, x+1] \models p$ (by (16)), iff $\lambda\left(l_{x}\right)=1$ (by (15) and (17)). So $\lambda\left(l_{x}\right)=w_{x}$. Hence, $D$ is a derivation tree for $w$ in $\mathcal{G}_{1}$. Since we can prove by the same argument that $w$ is generable also by $\mathcal{G}_{2}$, the languages generated by $\mathcal{G}_{1}$, and $\mathcal{G}_{2}$ both contain $w$, and thus the thesis.

Lemma 5 (completeness). Let $\mathcal{G}_{1}, \mathcal{G}_{2}$ be as in the previous lemma. If the languages generated by $\mathcal{G}_{1}, \mathcal{G}_{2}$ have a word in common, then the $L_{T}^{\pi}$-formula $\psi=\phi_{\mathcal{G}_{1}} \wedge \phi_{\mathcal{G}_{2}}$ is satisfiable in a model with frame of the form $\mathfrak{I n t}_{T}(T,<)$ for some finite strict linear order $(T,<)$.

Proof. Run Lemma 4's proof backwards: use derivation trees in $\mathcal{G}_{1}, \mathcal{G}_{2}$ for the common word to read off models of $\phi_{\mathcal{G}_{1}}, \phi_{\mathcal{G}_{2}}$ on the same base, on any $(T,<) \in$ Fin with long enough intervals.

Theorem 3. The logics $T^{\pi}$ (Fin) and $D^{\pi}($ Fin) are undecidable.

Proof. By [18, theorem 8.10], it is undecidable whether the languages generated by two context-free grammars have a common word. It is not hard to see that this is also true for context-free grammars in the form we have used. By Lemma 4 and 5 , this problem reduces to satisfiability of $L_{T}^{\pi}$-formulas over the intervals of finite linear orders, which is therefore also undecidable. The argument for $D^{\pi}$ (Fin) is symmetrical. 
The same argument shows that $D^{\pi}(\mathbb{N},<)$ and $T^{\pi}(\mathbb{N},>)$ are undecidable.

\section{Non-finite axiomatizability}

In this section we will show that the $\operatorname{logics} S(\mathcal{K})$ and $S^{\pi}(\mathcal{K})$ are not finitely axiomatizable using orthodox inference rules, for any non-empty $S \subseteq\{C, D, T\}$ and any class $\mathcal{K}$ with $(\mathbb{Q},<) \in \mathcal{K} \subseteq$ Lin. For example, $C D T^{\pi}(\operatorname{Lin})$ and $C D T^{\pi}$ (Dense) are not finitely axiomatizable. The argument is based on the so-called 'Monk algebras' from the theory of relation algebra (see, e.g., $[21,20,17])$, but we do not assume any knowledge of that topic here.

\subsection{Basic Definitions}

Let $\eta, \kappa>0$ be cardinals, and define an equivalence relation $\sim$ on $\eta \times \kappa=\{(i, j)$ : $i<\eta, j<\kappa\}$ by $(i, j) \sim\left(i^{\prime}, j^{\prime}\right)$ if and only if $j=j^{\prime}$. We think of $j$ as the color of $(i, j)$, so $\sim$ is the 'same color' relation. Then, we form a relational structure $\alpha(\eta, \kappa)$ as follows.

Definition 4. We define $\alpha(\eta, \kappa)$ as a tuple of the type $(I, \Pi, \rho)$, where:

- I $=(\eta \times \kappa) \cup\left\{1^{\prime}\right\}$, where $1^{\prime}$, called identity, is assumed not to be in $\eta \times \kappa$;

$-\Pi=\left\{1^{\prime}\right\}$

- $\rho$ is the set of all triples $(a, b, c) \in I^{3}$ such that 1$)$ one of $a, b, c$ is 1 ' and the other two are equal, or 2) $1^{\prime} \notin\{a, b, c\}$ and $a, b, c$ are not all $\sim$-equivalent.

The relations $R_{C}, R_{D}, R_{T} \subseteq I^{3}$ (for $I$ as above) are defined as follows:

$-R_{C}=\rho \backslash\left\{\left(1^{\prime}, a, a\right): a \in I, a \neq 1^{\prime}\right\}$,

$-R_{T}=\rho \backslash\left\{\left(a, 1^{\prime}, a\right): a \in I, a \neq 1^{\prime}\right\}$,

$-R_{D}=\rho \backslash\left\{\left(a, a, 1^{\prime}\right): a \in I, a \neq 1^{\prime}\right\}$.

We define the $S$-frame $\mathcal{F}_{S}(\eta, \kappa)=\left(I, \Pi, R_{\sharp}: \sharp \in S\right)$, for each $S \subseteq\{C, D, T\}$.

The $\mathcal{F}_{S}(\eta, \kappa)$ are 'non-standard' frames. They are not based on intervals, and indeed, this is detectable in $L_{S}$ : if $\eta, \kappa$ are finite and $\eta \gg \kappa$ then $\mathcal{F}_{S}(\eta, \kappa)$ does not even validate the logic $S(\operatorname{Lin})$. But $\mathcal{F}_{S}(\omega, \omega)$ is a bounded morphic image of $\mathfrak{I n t}(\mathbb{Q},<)$ (cf., e.g., [1, def. 2.10]), and so does validate $S^{\pi}(\mathcal{K})$. Since we can obtain $\mathcal{F}_{S}(\omega, \omega)$ as a 'limit' (via ultraproducts or compactness) of the finite frames $\mathcal{F}_{S}(\eta, \kappa)$, this will show that $S(\mathcal{K})$ and $S^{\pi}(\mathcal{K})$ are not finitely axiomatizable.

\subsection{Frames $\mathcal{F}_{S}(\eta, \kappa)$ not Validating $S($ Lin $)$}

Define $f: \omega \rightarrow \omega$ by $f(0)=1$, and $f(k+1)=1+(k+1) \cdot f(k)$ for every $k<\omega$. For any set $X$, and $1 \leq k<\omega$, a $k$-coloring of $X$ is a coloring of the edges of the complete undirected loop-free graph whose set of nodes is $X$, using at most $k$ colors, such that no 'monochromatic triangle' appears - that is, there is no 3-element subset of $X$ such that all three edges lying within it are given the same color. The following Ramsey-type theorem can be found in [15]. 
Proposition 1. Let $k \geq 1$. If a set $X$ has more than $f(k)$ elements then $X$ has no k-coloring.

Theorem 4. Suppose that $S \subseteq\{C, D, T\}$ is non-empty, $\eta>0, \kappa>1$, and $f(\kappa)<\left|\mathcal{F}_{S}(\eta, \kappa)\right|<\omega$. Then $\mathcal{F}_{S}(\eta, \kappa)$ does not validate $S($ Lin $)$.

Proof. One can write down a 'Jankov-Fine' formula $\phi$ (see, e.g., $[1, \S 3.4]$ ) describing $\mathcal{F}_{S}(\eta, \kappa)$ and satisfiable only in bounded morphic pre-images of $\mathcal{F}_{S}(\eta, \kappa)$. If $\mathcal{F}_{S}(\eta, \kappa)$ validated $S($ Lin $), \phi$ would be consistent with $S($ Lin $)$, and so there would be a bounded morphism $h$ from some concrete interval frame $\mathfrak{I n t}_{S}(T,<)$ onto $\mathcal{F}_{S}(\eta, \kappa)$. This would induce a partial $\kappa$-coloring of $T$ : the color of the edge $x y$ for $x<y$ would be the color of $h([x, y])$ when $h([x, y]) \neq 1$ '. As $\mathcal{F}_{S}(\eta, \kappa)$ is large, Lemma 1 shows that (assuming $C \in S$ ) some interval of $T$ would chop into two subintervals, all three having the same color. This contradicts the definition of $R_{C}$. The cases $D \in S, T \in S$ are similar.

\subsection{Frames $\mathcal{F}_{S}(\eta, \kappa)$ that Do Validate $S^{\pi}(\mathbb{Q},<)$}

We show here that $\mathcal{F}_{S}(\omega, \omega)$ does validate $S^{\pi}(\mathbb{Q},<)$, for any $S \subseteq\{C, D, T\}$. To do this, we will construct a bounded morphism from $\mathfrak{I n t}_{S}(\mathbb{Q},<)$ onto $\mathcal{F}_{S}(\omega, \omega)$ step by step, using the concepts of 'networks' and 'representations'.

Definition 5. A network is a map $N: B \times B \rightarrow \alpha(\omega, \omega)$, for some $B \subseteq \mathbb{Q}$, such that for all $x, y, z \in B$ we have:

1. $N(x, y)=1 \Longleftrightarrow x=y$,

2. $N(x, y)=N(y, x)$,

3. $(N(x, z), N(x, y), N(y, z)) \in \rho$.

We write $B(N)$ (the 'base' of $N)$ for the set $B=\{x \in \mathbb{Q}:(x, x) \in \operatorname{dom}(N)\}$. A network can be thought of as a complete directed graph with edges labeled by elements of $\alpha(\omega, \omega)$, satisfying the conditions above. As networks $N, M$ are functions, it follows from the definitions that $N \subseteq M$ iff $B(N) \subseteq B(M)$ and $N=M \uparrow(B(N) \times B(N))$.

Definition 6. A network $N$ is said to be a representation ${ }^{5}$ of $\alpha(\omega, \omega)$ if $B(N)=$ $\mathbb{Q}$ and the following hold for all $x, y \in \mathbb{Q}$, where the quantifiers range over $\mathbb{Q}$ :

1. $\exists z(z \leq x \wedge N(x, z)=a)$ and $\exists z(z \geq x \wedge N(x, z)=a)$ for every $a \in \alpha(\omega, \omega)$,

2. $x<y \rightarrow \exists z(x \leq z \leq y \wedge N(x, z)=a \wedge N(z, y)=b)$ whenever $(N(x, y), a, b) \in$ $\rho$,

3. $x<y \rightarrow \exists z(z \geq y \wedge N(x, z)=a \wedge N(z, y)=b)$ whenever $(N(x, y), a, b) \in \rho$ and $a \neq 1$

4. $x<y \rightarrow \exists z(z \leq x \wedge N(x, z)=a \wedge N(z, y)=b)$ whenever $(N(x, y), a, b) \in \rho$ and $b \neq 1$

${ }^{5}$ The term 'complete representation' would be more in accordance with usual algebraic terminology. 
Our aim is to show that $\alpha(\omega, \omega)$ has a representation. We can derive validity of $S^{\pi}(\mathbb{Q},<)$ in $\mathcal{F}_{S}(\omega, \omega)$ from this quite easily.

Lemma 6. $\alpha(\omega, \omega)$ has a representation.

Proof. We can build a representation by a 'step by step' game played on finite networks. In each round, one player challenges some failure of a property of Definition 6 for the current network. The other player replaces the network by an extension lacking this defect. This is always possible since we are using $\alpha(\eta, \kappa)$ with infinite $\kappa$, so that brand new colors can be used to label network edges whenever necessary. This avoids monochromatic triangles of the kind we met in Theorem 4. At the end of the game, a representation results.

Theorem 5. $\mathcal{F}_{S}(\omega, \omega)$ validates $S^{\pi}(\mathbb{Q},<)$ for any $S \subseteq\{C, D, T\}$.

Proof. A representation $N$ of $\alpha(\omega, \omega)$ induces a bounded morphism $h$ from $\mathfrak{I n t}_{S}(\mathbb{Q},<)$ onto $\mathcal{F}_{S}(\omega, \omega)$, via $h([x, y])=N(x, y)$. Bounded morphisms preserve validity of formulas.

\subsection{Non Finite Axiomatizability}

Theorem 6. For any non-empty $S \subseteq\{C, D, T\}$ and any class $\mathcal{K} \subseteq$ Lin that contains $(\mathbb{Q},<)$, the logics $S(\mathcal{K})$ and $S^{\pi}(\mathcal{K})$ are not finitely axiomatizable using standard inference rules (modus ponens, universal generalization, substitution).

Proof. Let $\Lambda$ be either one of $S(\mathcal{K}), S^{\pi}(\mathcal{K})$. Note that

$$
S(\operatorname{Lin}) \subseteq \Lambda \subseteq S^{\pi}(\mathbb{Q},<) .
$$

Assume for contradiction that $\Phi$ is a finite set of axioms for $\Lambda$ in its own signature $\left(L_{S}\right.$ or $\left.L_{S}^{\pi}\right)$. Then $(\bigwedge \Phi)=\phi\left(p_{1}, \ldots, p_{k}\right)$, say, axiomatises $\Lambda$ alone. For each finite $n>1$, choose finite $\eta_{n}$ so large that $\left|\alpha\left(\eta_{n}, n\right)\right|>f(n)$. By Theorem 4, $\mathcal{F}_{S}\left(\eta_{n}, n\right)$ does not validate even $S($ Lin), so by (18), it certainly does not validate $\Lambda$. Because the standard inference rules preserve validity over any frame, there is a model $\mathcal{M}_{n}=\left(\mathcal{F}_{S}\left(\eta_{n}, n\right), h_{n}\right)$ in which $\phi$ is not valid. We regard $\mathcal{M}_{n}$ as a first-order structure in an appropriate signature. Let $\mathcal{M}$ be a countable elementary substructure of a non-principal ultraproduct of the $\mathcal{M}_{n}$ (cf. [10]). By Loś's Theorem, it follows that $\mathcal{M} \cong \mathcal{F}_{S}(\omega, \omega)$, and that $\phi$ is not valid in $\mathcal{M}$. This contradicts Theorem 5 that $\mathcal{F}_{S}(\omega, \omega)$ validates $S^{\pi}(\mathbb{Q},<)$, and so (by $(18)$ ) certainly validates $\Lambda$.

The logics $C D T^{\pi}(\mathrm{Lin}), C D T^{\pi}(\mathrm{Dis}), C D T^{\pi}($ Dense $), C D T^{\pi}(\mathbb{Q})$ were finitely axiomatized by Venema [25], using an unorthodox Burgess/Gabbay-style inference rule. There is no conflict between this result and Theorem 6. A formula derived from a formula valid in $\mathcal{F}_{S}(\eta, \kappa)$ using Venema's rule need not be valid in $\mathcal{F}_{S}(\eta, \kappa)$. So the argument of Theorem 6 fails when this rule is added. 


\begin{tabular}{|l|l|l|l|l|l|l|}
\hline \multicolumn{1}{|c|}{$\mathcal{K}$} & \multicolumn{1}{c|}{$C(\mathcal{K})$} & \multicolumn{1}{c|}{$C^{\pi}(\mathcal{K})$} & \multicolumn{1}{c|}{$D(\mathcal{K})$} & \multicolumn{1}{c|}{$D^{\pi}(\mathcal{K})$} & \multicolumn{1}{c|}{$T(\mathcal{K})$} & \multicolumn{1}{c|}{$T^{\pi}(\mathcal{K})$} \\
\hline Lin & Und., NFA & Und.[13],NFA & Und., NFA & Und., NFA & Und., NFA & Und., NFA \\
Asc & Und., NFA & Und., NFA & NFA & NFA & Und., NFA & Und., NFA \\
Des & Und., NFA & Und., NFA & Und.,NFA & Und., NFA & NFA & NFA \\
Fin & $?$ & Und.[22] & $?$ & Und. & $?$ & Und. \\
Dense & Und., NFA & Und.[19],NFA & Und., NFA & Und., NFA & Und., NFA & Und., NFA \\
Dis & Und. & Und. & Und. & Und. & Und. & Und. \\
$\mathbb{N}$ & $?$ & Und.[22], & $?$ & Und. & Und. & Und. \\
$\mathbb{Z}$ & $?$ & Und.[22], & Und. & Und. & Und. & Und. \\
$\mathbb{Q}$ & Und., NFA & Und.[19],NFA & Und., NFA & Und, NFA & Und., NFA & Und., NFA \\
\hline
\end{tabular}

Table 1. A resume of the results of this paper.

\section{Conclusions}

In this paper we have considered the temporal logic for intervals CDT introduced by Venema in [25]. Finite axiomatizability with standard rules and decidability/undecidability of CDT itself and of its possible single-modality fragment is a natural question that was open since the introduction of the logic, and to which we have answered here. Results are summarized in Table 1, where 'Und.' means undecidable and 'NFA' indicates non-finite axiomatizability.

Acknowledgments. Angelo Montanari was co-financed by the Italian national project on "Constraint and prefereneces as unifying formalism for system analysis and solutions of real problems", and Guido Sciavicco was co-financed by the Spanish MEC - FEDER project "IDEATIO", Ref. No: TIN2006-15460-C04-01.

\section{References}

1. P. Blackburn, M. de Rijke, and Y. Venema. Modal Logic. Cambridge University Press, 2002.

2. E. Börger, E. Grädel, and Y. Gurevich. The Classical Decision Problem. Perspectives of Mathematical Logic. Springer, 1997.

3. H. Bowman and S. Thompson. A decision procedure and complete axiomatization of finite interval temporal logic with projection. Journal of Logic and Computation, 13(2):195-239, 2003.

4. D. Bresolin, V. Goranko, A. Montanari, and P. Sala. Tableau-based Decision Procedure for the Logic of Proper Subinterval Structures over Dense Orderings. In C. Areces and S. Demri, editors, Proceedings of M4M-5: 5th International Workshop on Methods for Modalities, pages 335-351, 2007.

5. D. Bresolin, V. Goranko, A. Montanari, and P. Sala. Tableau Systems for Logics of Subinterval Structures over Dense Orderings. In N. Olivetti, editor, Proc. of TABLEAUX 2007, volume 4548 of LNAI, pages 73-89. Springer, 2007.

6. D. Bresolin, V. Goranko, A. Montanari, and G. Sciavicco. On Decidability and Expressiveness of Propositional Interval Neighborhood Logics. In Proc. of the International Symposium on Logical Foundations of Computer Science (LFCS), volume 4514 of $L N C S$, pages 84-99. Springer, 2007. 
7. D. Bresolin, V. Goranko, A. Montanari, and G. Sciavicco. Propositional interval neighborhood logics: Expressiveness, decidability, and undecidable extensions. Technical Report 05, Department of Mathematics and Computer Science, University of Udine, Italy, 2008.

8. D. Bresolin, A. Montanari, and P. Sala. An optimal tableau-based decision algorithm for Propositional Neighborhood Logic. In Proceedings of STACS 200\%, volume 4393 of LNCS, pages 549-560. Springer, 2007.

9. D. Bresolin, A. Montanari, and G. Sciavicco. An optimal decision procedure for Right Propositional Neighborhood Logic. Journal of Automated Reasoning, 38(13):173-199, 2007.

10. C. C. Chang and H. J. Keisler. Model theory. North-Holland, Amsterdam, 1990.

11. D.M. Gabbay. An irreflexive lemma with applications to axiomatization on linear frames. Aspects of Philosophical Logic, pages 67-89, 1981.

12. V. Goranko, A. Montanari, and G. Sciavicco. Propositional interval neighborhood temporal logics. Journal of Universal Computer Science, 9(9):1137-1167, 2003.

13. V. Goranko, A. Montanari, and G. Sciavicco. A road map of interval temporal logics and duration calculi. J. of Applied Non-Classical Logics, 14(1-2):9-54, 2004.

14. V. Goranko, A. Montanari, G. Sciavicco, and P. Sala. A general tableau method for propositional interval temporal logics: Theory and implementation. Journal of Applied Logic, 4(3):305-330, 2006.

15. R. E. Greenwood and A. M. Gleason. Combinatorial relations and chromatic graphs. Canadian Journal of Mathematics, 7:1-7, 1955.

16. J. Halpern and Y. Shoham. A propositional modal logic of time intervals. Journal of the ACM, 38(4):935-962, 1991.

17. R Hirsch and I Hodkinson. Relation algebras by games, volume 147 of Studies in Logic and the Foundations of Mathematics. North-Holland, Amsterdam, 2002.

18. J. E. Hopcroft and J. D. Ullman. Introduction to automata theory, languages, and computation. Addison-Wesley, 1979.

19. K. Lodaya. Sharpening the undecidability of interval temporal logic. In Proc. of 6th Asian Computing Science Conference, volume 1961 of LNCS, pages 290-298. Springer, 2000.

20. R. Maddux. Relation algebras, volume 150 of Studies in Logic and the Foundations of Mathematics. Elsevier, Amsterdam, 2006.

21. J. D. Monk. Nonfinitizability of classes of representable cylindric algebras. Journal Symbolic Logic, 34:331-343, 1969.

22. B. Moszkowski. Reasoning about digital circuits. Tech. rep. stan-cs-83-970, Dept. of Computer Science, Stanford University, Stanford, CA, 1983.

23. S. Roy and G. Sciavicco. Completeness of chop. In H. W. Guesguen, G. Ligozat, and R.V. Rodriguez, editors, Proc. IJCAI-07, pages 90-95, 2007.

24. Y. Venema. Expressiveness and completeness of an interval tense logic. Notre Dame Journal of Formal Logic, 31(4):529-547, 1990.

25. Y. Venema. A modal logic for chopping intervals. Journal of Logic and Computation, 1(4):453-476, 1991. 